\title{
Echo suppression and discrimination suppression aspects of the precedence effect
}

\author{
XUEFENG YANG and D. WESLEY GRANTHAM \\ Vanderbilt University Medical Center, Nashville, Tennessee
}

\begin{abstract}
The precedence effect is a phenomenon that may occur when a sound from one direction (the lead) is followed within a few milliseconds by the same or a similar sound from another direction (the lag, or the echo). Typically, the lag sound is not heard as a separate event, and changes in the lag sound's direction cannot be discriminated. The hypothesis is proposed in this study that these two aspects of precedence (echo suppression and discrimination suppression) are at least partially independent phenomena. Two experiments were conducted in which pairs of noise bursts were presented to subjects from two loudspeakers in the horizontal plane to simulate a lead sound and a lag sound (the echo). Echo suppression threshold was measured as the minimum echo delay at which subjects reported hearing two sounds rather than one sound; discrimination suppression threshold was measured as the minimum echo delay at which subjects could reliably discriminate between two positions of the echo. In Experiment 1, it was found that echo suppression threshold was the same as discrimination suppression threshold when measured with a single burst pair (average 5.4 msec). However, when measured after presentation of a train of burst pairs (a condition that may produce "buildup of suppression"), discrimination suppression threshold increased to $10.4 \mathrm{msec}$, while echo suppression threshold increased to $26.4 \mathrm{msec}$. The greater buildup of echo suppression than of discrimination suppression indicates that the two phenomena are distinct under buildup conditions and may be the reflection of different underlying mechanisms. Experiment 2 investigated the effect of the directional properties of the lead and lag sounds on discrimination suppression and echo suppression. There was no consistent effect of the spatial separation between lead and lag sources on discrimination suppression or echo suppression, nor was there any consistent difference between the two types of thresholds (overall average threshold was $5.9 \mathrm{msec}$ ). The negative result in Experiment 2 may have been due to the measurements being obtained only for single-stimulus conditions and not for buildup conditions that may involve more central processing by the auditory system.
\end{abstract}

In a reflective environment, such as a normal-sized room, listeners are generally able to localize a sound source at the correct direction despite the presence of echoes arriving from the walls, ceiling, and floor. The directional information of the first arriving sound at the ears dominates the perception of the location of the sound source, whereas the directional information carried by the reflections arriving only a few milliseconds later is largely suppressed. This phenomenon is known as the precedence effect, or the law of the first wavefront (Wallach, Newman, \& Rosenzweig, 1949; Zurek, 1987).

This research was supported by NIH Grant DC00185. It forms a portion of the dissertation completed by the first author in partial fulfillment of the requirements for the doctoral degree in the Division of Hearing and Speech Sciences at Vanderbilt University, 1995. Some of the results were reported at the 129th Meeting of the Acoustical Society of America, May 1995 (Yang \& Grantham, 1995). The authors are grateful to Ruth Litovsky and three anonymous reviewers for many helpful comments on earlier versions of this manuscript. The authors also thank Don Riggs for assistance in the preparation of figures. Correspondence should be addressed to D. W. Grantham, Vanderbilt Bill Wilkerson Center for Otolaryngology and Communication Sciences, 1114 19th Ave. South, Nashville, TN 37212 (e-mail: granthdw@ctrvax.vanderbilt.edu).

\section{Measures of Precedence}

In order to appreciate the various aspects of the precedence effect, consider the simple situation illustrated in Figure 1, in which a direct sound is presented from one direction in the horizontal plane (shown as A), and a single reflection produced by a wall arrives from a different direction $\left(A^{\prime}\right)$. In this case, the position (angle) of the wall relative to the listener determines the direction of the reflection, and the distance of the wall determines the delay of the reflection (the echo delay). The tasks that have typically been employed to measure the dominance of the first arriving sound may be classified into three categories.

(1) Fusion, or echo suppression: The subject is asked to state how many events were perceived. For very short echo delays, subjects report "one event" (i.e., the two sounds are fused; the echo is "suppressed"). For long echo delays, they report "two events" (i.e., the two sounds are not fused; the echo is not suppressed). The critical echo delay corresponding to the transition between "one" and "two" is called echo threshold (Blauert, 1983, pp. 222-235; Clifton \& Freyman, 1989; Freyman, Clifton, \& Litovsky, 1991). Experiments reported to date in this category have been conducted with free-field stimuli. (2) Localization dominance: The subject is asked to localize the "com- 


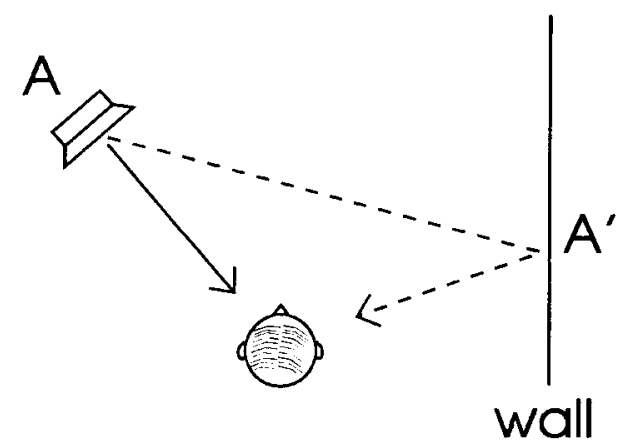

Figure 1. Schematic diagram of acoustic information arriving at a subject when a direct sound is presented in the presence of a single reflecting surface (the wall). The direct sound arrives from Position $A$, and the single reflection arrives a few milliseconds later from Position A'.

posite" (fused) image produced by the combined source and reflection; to the extent that the subject points to the source position at $\mathrm{A}$, the first arriving sound dominates the perception of location. In one version of this task, the echo direction and echo delay are held constant, and the subject must adjust the position of the direct sound so that the apparent location of the composite image matches a designated position (e.g., midline). Such "adjustment" experiments (Shinn-Cunningham, Zurek, \& Durlach, 1993; Wallach et al., 1949; Yost \& Soderquist, 1984) have employed headphones and have had subjects adjust the lateral position of images (simulated direct sound and echo) within the head. In another version of this task, Hafter, Saberi, Jensen, and Briolle (1992) had subjects point to the apparent location of the fused image in a free-field setting, such as that shown in Figure 1. (3) Lag discrimination: The subject must discriminate between two possible directions of the lag sound. Since the discrimination of the lag sound's position is typically worse in the presence of the lead sound than in its absence, we may refer to the suppression of lag discrimination produced by the lead sound, or, more briefly, to discrimination suppression. Experiments in this category have been conducted both with headphones (Saberi \& Perrott, 1990; Shinn-Cunningham et. al., 1993; Zurek, 1980 ) and in the free field (Freyman et al., 1991; Hafter et al., 1992; Litovsky \& Macmillan, 1994; Perrott, Marlborough, Merrill, \& Strybel, 1989).

Experiments in the present study will be concerned with the first and third of these tasks-that is, with echo suppression and discrimination suppression.

\section{The Relationship Among \\ Measures of Precedence}

An important question in the study of the precedence effect is whether it is a single phenomenon, mediated by a single underlying mechanism (which appears to be the typical view in the literature), or whether it may encompass multiple processes. One approach to this question would be to compare performance in the three tasks described above as a function of common variables in order to ascertain whether functional relationships for the different measures coincide or diverge from one another. To date, there has been little systematic comparison of performance in the three tasks; two notable exceptions are the studies of Shinn-Cunningham et al. (1993) and Freyman et al. (1991).

Shinn-Cunningham et al. (1993) obtained adjustment (localization) and lag discrimination measures in the same subjects from precedence-effect stimuli (noise bursts) presented over headphones. They successfully modeled the results from both experiments (and from other adjustment and discrimination experiments in the literature) within a single framework that quantified the relative weights given to the lead and lag (echo) events as a function of echo delay and the "direction" of the lead and lag stimuli. These results suggest that localization dominance and discrimination tasks (the second and third measures in the classification scheme outlined above) may indeed reflect the operation of a single underlying mechanism, at least for headphone-presented stimuli.

The second study that has directly compared performance in different tasks (Freyman et al., 1991) measured echo suppression (fusion) and discrimination suppression in the same subjects (the first and third tasks in the classification). Using 4-msec noise burst stimuli, these investigators modified the usual discrimination paradigm: Instead of holding echo delay constant and varying the angle of the reflection to obtain a "minimum audible angle" for the echo (see Litovsky \& Macmillan, 1994; Perrott et al., 1989), Freyman et al. (1991) held the angular distance between two echo positions constant (at $20^{\circ}$ ) and varied the echo delay to obtain a critical delay at which the angular discrimination could just be made. In this way, they were able to compare the echo threshold for fusion (that delay at which subjects could just hear "two events") directly with the echo threshold for discrimination (that delay at which subjects could just discriminate a $20^{\circ}$ shift in echo position). For purposes of the present paper, we will henceforth refer to these two measures as echo suppression threshold and discrimination suppression threshold, respectively. ${ }^{1}$

Three of the 4 subjects in Freyman et al.'s experiment showed approximately the same discrimination suppression threshold as echo suppression threshold (between 8 and $12 \mathrm{msec}$ ); the 4 th subject had a lower echo suppression threshold $(7 \mathrm{msec})$ than discrimination suppression threshold $(12 \mathrm{msec})$. These results were interpreted by the authors as evidence that the two tasks were likely measuring the same phenomenon.

The combined results from Shinn-Cunningham et al. (1993) and Freyman et al. (1991) suggest that all three measures of precedence outlined above reflect the operation of a single underlying mechanism. If this is the case, the degree to which the lead sound dominates in a 
localization task should be highly correlated with the degree to which information about the echo's position is suppressed and the degree to which the echo's identity as a separate event is suppressed.

The apparent coincidence among the three measures of precedence in the studies of Freyman et al. (1991) and Shinn-Cunningham et al. (1993) was based on data obtained in "baseline" conditions, in which only a single stimulus pair was presented to subjects on each trial. However, there is evidence that the precedence effect can be significantly enhanced if repeated stimulation is provided over a long time interval (measured in seconds). The question arises whether the measures of precedence will be affected in the same way under conditions of repeated stimulation.

\section{Buildup of Suppression}

A few investigators (Clifton, 1987; Clifton \& Freyman, 1989; Clifton, Freyman, Litovsky, \& McCall, 1994; Freyman et al., 1991; Thurlow \& Parks, 1961; Yang \& Grantham, 1993, 1994) have employed stimulus trains to study dynamic aspects of echo suppression and discrimination suppression. These studies reported that a "buildup" of suppression could be produced over the course of an extended train of clicks; in other words, the amount of echo suppression and/or discrimination suppression appeared to increase with extended or repeated stimulation from a particular source. (To date, there have been no published reports on the effects of repeated stimulation on localization dominance.) Clifton et al. (1994) hypothesized that buildup of suppression is brought about by subjects' changing expectations about the acoustics of a particular room based on their auditory experience in that room; thus, the longer a person remains in a normal room (over time intervals measured in seconds), the more the auditory system suppresses information about the acoustic reflections. These results suggest that the precedence effect should be conceptualized as a dynamic process that is influenced by previous and ongoing stimulation (Clifton \& Freyman, 1989; Clifton et al., 1994).

Not only does the amount of suppression increase in most subjects under conditions of repeated stimulation, but it has been shown that certain features of suppression are revealed under buildup conditions that are not apparent under baseline conditions. For example, Grantham (1996) showed that discrimination suppression threshold is asymmetric (with more suppression occurring for most subjects when the direct sound comes from the subject's right side than when it comes from the left side), but that this asymmetry occurs only under buildup conditions. These results suggest that the effects observed in buildup conditions may involve more central mediation than that observed with simple baseline presentation.

During the course of some previous work on the precedence effect (Yang \& Grantham, 1993, 1994), ${ }^{2}$ we serendipitously observed an unexpected and somewhat counterintuitive result: Echo suppression appeared to be greater than discrimination suppression under buildup condi- tions. In other words, a listener would sometimes report hearing only one event, and yet, if forced to make a discrimination decision about the echo, he or she would be able to respond above chance. Such a result suggests that the precedence effect may not be the result of a single auditory process.

In order to confirm these informal observations, Experiment 1 was designed to systematically compare echo suppression and discrimination suppression under baseline and buildup conditions.

\section{Spatial Tuning of the Precedence Effect}

A second purpose of this investigation was to measure echo suppression and discrimination suppression as a function of the spatial separation between lead and lag sources. To date, there have been only a few scattered allusions in the literature to the effects on precedence of lead-lag spatial separation. Some localization dominance experiments have reported that dominance of the lead event in a lateralization task (these studies employed headphones) increased as the spatial separation between direct and reflected sounds increased (the adjustment experiments of Shinn-Cunningham et al., 1993, Wallach et al., 1949, and Yost \& Soderquist, 1984). Likewise, in a discrimination suppression experiment, Litovsky, Dizon, Pazmany, and Colburn (1996) found that suppression increased as the lead-lag separation increased (for virtual-field horizontal-plane sources). On the other hand, the opposite result has been reported in some fusion experiments: Echo suppression increased as the separation between direct and reflected sound decreased (Boerger, as reported in Blauert, 1983; Hafter et al., 1992; Yost \& Guzman, 1995).

From these scattered reports, it appears that discrimination suppression increases while echo suppression decreases as the directions of the lead and lag sources diverge. Such a result would lend further support to the relative independence of discrimination suppression and echo suppression aspects of the precedence effect. Experiment 2 was designed to test the hypothesis that the two types of suppression are tuned differently with respect to lead-lag spatial separation.

\section{EXPERIMENT 1}

\section{Method}

Subjects. Six adults ( 2 males, 4 females; $23-38$ years of age) with normal hearing in both ears were recruited to participate in this experiment. All subjects were paid, and none was familiar with the purposes and hypotheses of the study. The subjects were tested individually in sessions that lasted $1-2 \mathrm{~h}$. The subjects were given frequent breaks during each session to avoid fatigue.

The first two to four sessions for each subject were considered practice, and the data from these sessions were not included in the later analyses; subsequent inspection of the data indicated that performance had stabilized by this time.

Apparatus and Stimuli. The experiment was conducted in a $6 \times 6 \times 6 \mathrm{~m}$ anechoic chamber lined on all six surfaces with $0.75-\mathrm{m}$ foam wedges. During a session, the subject was seated in a chair at the center of the chamber. The subject's head was not re- 


\section{Preceding Train of Bursts}
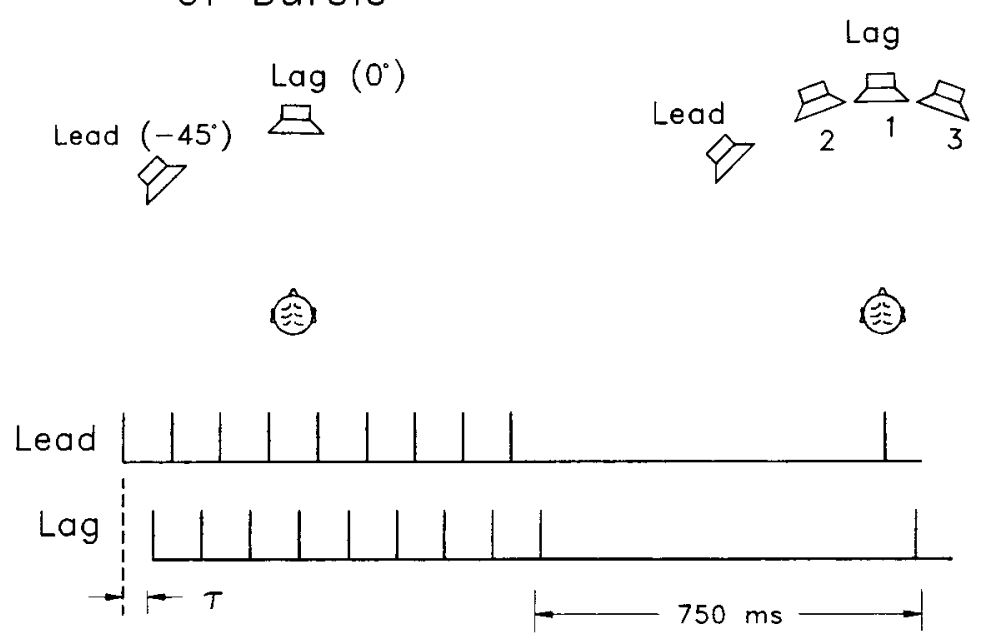

Figure 2. Loudspeaker arrangement and stimulus timing employed in Experiment 1 . In the buildup conditions, each trial began with a train of 9 burst pairs, with the lead burst coming from the loudspeaker at $-45^{\circ}$ and the lag burst coming from the loudspeaker at $0^{\circ}$ (delay between lead and lag bursts was $\tau \mathrm{msec}$ ). For the test burst pair, the lead burst came from the loudspeaker at $-45^{\circ}$, and the lag burst came from Loudspeaker 1 at $0^{\circ}$ in the echo suppression task or from one of the flanking loudspeakers (Loudspeaker 2 at $-5^{\circ}$, or Loudspeaker 3 at $+5^{\circ}$ ) in the discrimination suppression task. In the baseline conditions, the preceding train of burst pairs was not presented; only the test burst pair was presented. strained, but she or he was instructed to face directly ahead and not move the head during the run. A semicircular array of loudspeakers (JBL $8110 \mathrm{H}$ high-compliance full-range) was positioned in front of the subject at ear level, $1.8 \mathrm{~m}$ in distance. From this array, four loudspeakers were employed in Experiment 1. The room lights were extinguished in order to eliminate possible visual influences on performance.

Stimuli were 4-msec bursts of Gaussian noise, output at a 20 $\mathrm{kHz}$ rate through a two-channel 12-bit DAC and bandpass-filtered from 100 to $10000 \mathrm{~Hz}$. The spectrum of the resulting bursts had a gradual $(5 \mathrm{~dB})$ rolloff between $250 \mathrm{~Hz}$ and $10 \mathrm{kHz}$, followed by a steep rolloff ( $126 \mathrm{~dB} /$ octave) above $10 \mathrm{kHz}$. Bursts were always presented in pairs: one from a lead loudspeaker and one from a lag loudspeaker. The two bursts within a pair were identical temporal waveforms with a specified delay between lead and lag; each new burst pair was unique, generated from a Gaussian random number generator at run time. Level of the bursts (calibrated separately for each loudspeaker) was set such that the average intensity was $65 \mathrm{dBA}$ when played continuously and measured at the position of the subject's head.

Two measures of performance were obtained: discrimination suppression threshold and echo suppression threshold (described below). For each of these tasks, threshold was obtained in two conditions: (1) a baseline condition in which the subjects made judgments about a single burst pair, and (2) a "buildup" condition in which the subjects made judgments about a test burst pair that followed a train of nine preceding burst pairs. Thus, a total of four types of threshold were obtained: echo suppression and discrimination suppression thresholds in both baseline and buildup conditions. Order of the four conditions was random and counterbalanced across subjects.

Loudspeaker configuration and timing sequence are shown in Figure 2, which will be referred to in the following paragraphs.
Measurement of discrimination suppression threshold. For the measurement of discrimination suppression in the buildup condition, a train of nine pairs of noise bursts was presented to begin each trial (at a rate of 4 bursts/sec), with the lead burst from a loudspeaker at $-45^{\circ}$ and the lag burst from a loudspeaker at $0^{\circ}$ azimuth (see left side of Figure 2). Following a delay of $750 \mathrm{msec}$, a test burst pair was presented, in which the lead burst came from the same loudspeaker as the lead burst during the train $\left(a t-45^{\circ}\right.$ ), and the lag burst came from one of the two loudspeakers flanking the midline loudspeaker (Loudspeaker 2 at $-5^{\circ}$ or Loudspeaker 3 at $+5^{\circ}$, see right side of Figure 2). The subject's task was to choose which lag loudspeaker was activated, using a lap-held response box. Immediate feedback was given by LEDs on the response box.

Echo delay ( $\tau$ in Figure 2), which was always the same on any given trial for the train burst pairs and the test burst pair, was varied adaptively to track threshold. Each adaptive block began with an initial echo delay of $60 \mathrm{msec}$ between the lead and lag sounds (a condition that the subjects found easy). After two correct responses in a row, echo delay decreased (by a factor of 1.4); after each incorrect response, echo delay increased by this factor. The block terminated after 10 reversals of echo delay change, and discrimination suppression threshold ( $70.7 \%$ correct discrimination level) was computed as the mean of the last 8 of these reversals (Levitt, 1971). If 10 reversals had not occurred within 50 trials, the block terminated and the run was discarded (this rarely happened).

Discrimination suppression threshold in the baseline condition was obtained in a similar fashion, except there was no leading train; each trial consisted of only a single test burst pair (as shown on the right side of Figure 2), and the subject had to respond whether the lag burst came from Loudspeaker 2 or 3 .

For both baseline and buildup conditions, at least four thresholds (i.e., four runs) were obtained from each subject. If the standard deviation for a run (calculated across reversal points) was 
test trial

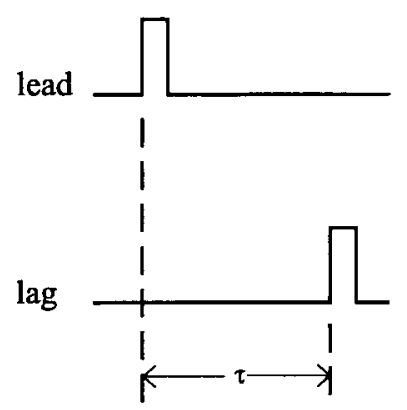

echo delay

(adaptively varied) catch trial

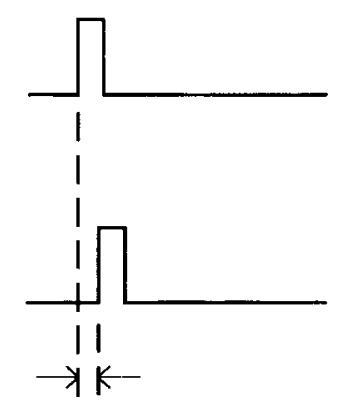

echo delay $(1.5-1.8 \mathrm{~ms})$

Figure 3. Timing sequence for test trials and for catch trials in the measurement of echo suppression threshold. A lead and lag stimulus was presented on each trial; however, for the catch trials, the echo delay was well below echo threshold, so it was always identified as "one event."

greater than one third of the threshold magnitude, that run was discarded. If the standard deviation calculated across the four thresholds was greater than one third of the average of the four thresholds, two more thresholds were obtained for that condition, and all six were included in the final analysis. Additional threshold runs were obtained in about $50 \%$ of the cases.

Measurement of echo suppression threshold. The sequence of stimulus presentation for the measurement of echo suppression was very similar to that described above for discrimination suppression. In the buildup condition, each trial began with the train of 9 burst pairs (presented at a rate of $4 / \mathrm{sec}$ ) as shown on the left side of Figure 2. Following a delay of $750 \mathrm{msec}$, a test burst pair was presented, for which the lead and lag bursts came from the same loudspeakers that had produced them during the train (the lag sound came from Loudspeaker 1, as shown on the right side of Figure 2). In this case, the subject's task was to report whether she or he heard one or two sounds in the test burst (i.e., was the echo event heard as separate?). Feedback was not presented (unbeknownst to the subject, two sounds were always presented-one from the lead and one from the lag speaker).

As described above for discrimination suppression thresholds, echo delay was always the same within a given trial for both train bursts and the test burst and was varied adaptively to track a threshold. The initial delay was sufficiently large $(30-90 \mathrm{msec})$ so that all subjects would clearly hear two events. After two successive responses of "two events," echo delay decreased (by a factor of 1.4); after each response of "one event," echo delay increased by this factor. Echo suppression threshold $(70.7 \%$ correct discrimination level) was calculated from the last 8 of 10 reversals of echo delay change, as described earlier for discrimination suppression threshold (Levitt, 1971).

A feature that was different for the measurement of echo suppression threshold from that described earlier for discrimination suppression threshold was the inclusion of "catch trials" among the "standard trials" just described (see Figure 3). Echo delay in the catch trials was set well below echo threshold (randomized between 1.5 and $1.8 \mathrm{msec}$ ), and these trials were always perceived as "one event." Catch trials were employed in an attempt to obtain a more objective measure of echo suppression than that obtained in previous studies. ${ }^{3}$ The proportion of catch trials was .5 at the beginning of a run but was reduced to .25 after the first 2 reversals in the adaptive tracking procedure, since the lower proportion proved in preliminary testing to be more efficient for threshold determi-

nation. The adaptive tracking was carried out on the basis of the responses to the standard trials alone and not to the catch trials.

The measurement of echo suppression in the baseline condition was the same as that just described for the buildup condition, except there was no leading train: Each trial consisted of a single test burst, to which the subject had to respond "one" or "two."

At least four thresholds were obtained from each subject in both baseline and buildup conditions, with the same rules for inclusion as described earlier for discrimination suppression thresholds.

\section{Results and Discussion}

Echo suppression thresholds and discrimination suppression thresholds for each of the 6 subjects in the two conditions are displayed in Figure 4, along with the mean across subjects (lower right panel). In the baseline (without train) conditions, average discrimination suppression threshold and echo suppression threshold were 5.6 and $5.2 \mathrm{msec}$, respectively. In the buildup (with train) conditions, average thresholds increased to 10.4 and $26.4 \mathrm{msec}$, respectively.

A $2 \times 2$ analysis of variance (ANOVA) was performed on the subjects' mean thresholds, with condition (baseline and buildup) and type of suppression (echo suppression and discrimination suppression) as withinsubjects factors. Results of the ANOVA and follow-up post hoc tests confirmed the patterns shown in Figure 4. First, there was a significant interaction between condition and type of suppression $[F(1,5)=12.33, p<.017]$. Second, in the baseline condition, there was no significant difference between echo suppression threshold and discrimination suppression threshold $[F(1,5)=0.84]$, thus replicating the findings of Freyman et al. (1991). Third, the train produced a significant threshold elevation for both echo suppression threshold $[F(1,5)=19.78, p<$ $.001]$ and discrimination suppression threshold $[F(1,5)$ $=8.11, p<.05]$. Finally, as predicted, there was a significant difference between echo suppression and discrimination suppression in the buildup conditions $[t=$ $3.71, p<.014]$.

Is there a facilitative effect of the train on discrimination performance? Despite considerable intersubject variability, echo suppression thresholds were consistently larger than discrimination suppression thresholds under buildup conditions (Figure 4). Prior to considering possible implications of this result for precedence effect mechanisms, we consider some features of the stimulus and task paradigm that may have contributed to this difference. In particular, it is possible that the presentation of the preceding train of bursts actually served to facilitate performance in the discrimination task, such that even though the preceding train generally produced an increase in both types of suppression, the increase in discrimination suppression was less than it would have been if this facilitation effect had not occurred. Such a relative facilitative effect on discrimination performance might have occurred, for example, because the repeated bursts in the train may have served to stabilize the subject's internal representation of the burst pair's composite location or percept, thus rendering the subsequent discrimination of the test burst echo position relatively easier. 


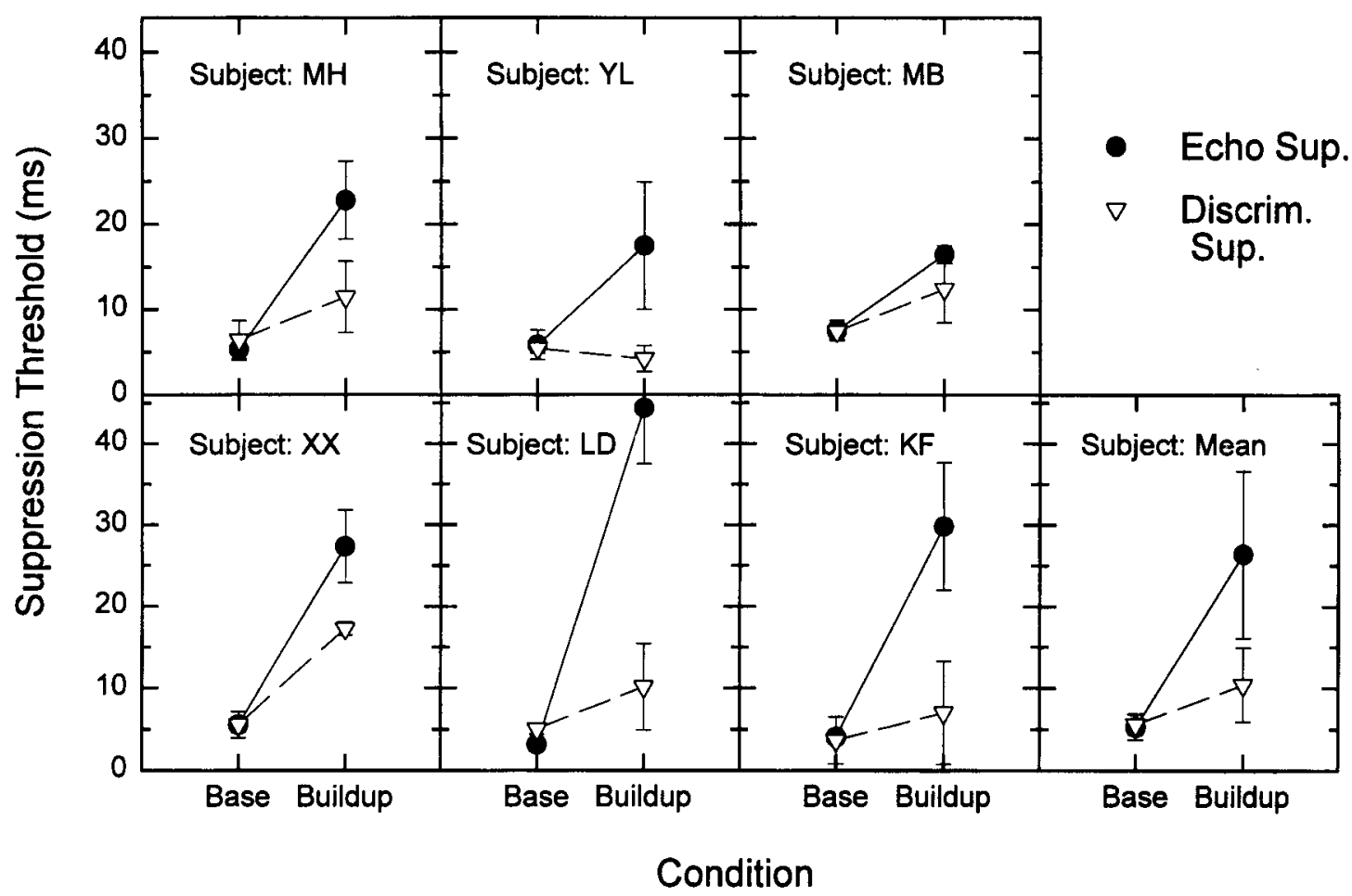

\begin{abstract}
Figure 4. Echo suppression thresholds (filled circles) and discrimination suppression thresholds (open triangles) for the baseline condition (without preceding train) and the buildup condition (with preceding train). Data are shown for the 6 subjects in different panels (means across subjects are shown in the far right panel). Error bars show \pm 1 standard deviation computed across runs for the individual subjects or computed across subjects for the mean data (in the far right panel).
\end{abstract}

Another possibility is that the $\pm 5^{\circ}$ shift in the echo position for the test burst presentation actually served to release some of the buildup achieved during the train, thus restoring some discriminability (Clifton et al., 1994, showed that a change in echo delay after a preceding train-simulating a sudden change in the position of a reflecting wall-could restore discriminability to near baseline levels).

These types of facilitative effects suggest that the buildup discrimination suppression thresholds shown in Figure 4 may be underestimates of the true degree of discrimination suppression. It is difficult from the present data to assess the extent of such underestimation; however, there is some indirect evidence suggesting that the degree of underestimation of these thresholds is small, at best, and therefore does not affect the general pattern of data shown in Figure 4. With respect to the first factor (stabilization of the internal representation), the effect of stimulus duration on horizontal-plane localization accuracy is minimal, even for presentations down to $5 \mathrm{msec}$ (Rakerd \& Hartmann, 1986), suggesting that stabilization of the internal image with repeated bursts would not show a large effect. With respect to the second factor (release of buildup), the $\pm 5^{\circ}$ shift in echo position employed in the present experiment is much smaller than the changes found by Clifton et al. (1994, a shift in depth of $1-2 \mathrm{~m}$ ) to produce significant restoration of discrim- ination threshold. In short, we feel the differences in buildup thresholds for echo suppression and discrimination suppression, although perhaps exaggerated somewhat due to the factors mentioned in the previous paragraph, are real differences that reflect the operation of the precedence effect. ${ }^{4}$

Implications for mechanisms underlying the precedence effect. The results shown in Figure 4 suggest that when a single burst pair is presented in isolation, discrimination suppression and echo suppression reflect the processing of a common mechanism (possibly of relatively peripheral origin). However, with more extended or repeated presentation of stimuli (as would occur when an observer gains acoustic experience in a room), a higher level processing might be invoked that serves to reduce the load from incoming signals that can be identified as echoes (and, thus, as unimportant or confusing signals). Both types of suppression increase under these circumstances, but the larger increase in echo than in discrimination suppression indicates that an echo's identity as a separate event is the first thing suppressed. The fact that an echo's direction can be discriminated over a range of echo delays for which it is not identifiable as a separate event (when presented from midline) supports the conclusion that echo suppression and discrimination suppression are distinctive phenomena, at least under buildup conditions. It also indicates that "echo suppres- 

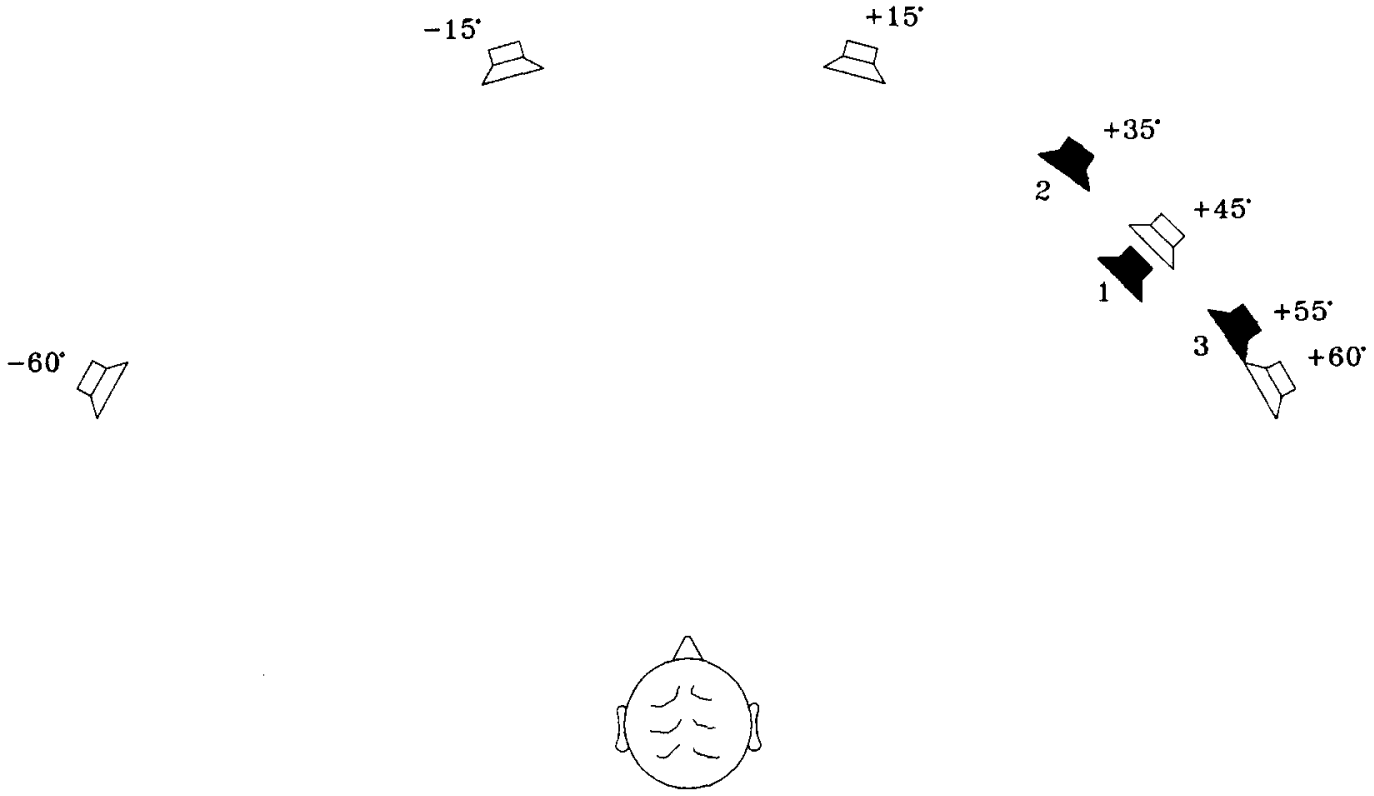

Figure 5. Loudspeaker configuration employed in Experiment 2. Lead source position was at one of the five azimuths shown by the open loudspeaker symbols. Lag source position, shown by the filled loudspeaker symbols, was constant at $+45^{\circ}$ azimuth for the echo suppression task or at one of the flanking positions, 2 or 3 , for the discrimination suppression task.

sion" by no means implies that all information concerning the echo is necessarily unavailable to the listener (see Saberi \& Perrott, 1990): When a subject's attention is specifically drawn to the echo event, as it was in the present experiment, there is a range of echo delay (10-20 msec for our subjects) for which the echo's direction can be discriminated even when it is "suppressed."

In terms of the different aspects of precedence outlined in the introduction, the present data, together with the data and analysis of Shinn-Cunningham et al. (1993), are consistent with the following scenario: Under baseline conditions, the three aspects of precedence are mediated by a common mechanism; however, under buildup conditions, echo suppression and discrimination suppression are mediated by distinct mechanisms. To date, there are no published data reporting whether localization dominance shows any change under buildup conditions.

\section{EXPERIMENT 2}

Experiment 2 was designed to measure echo suppression and discrimination suppression as a function of the azimuthal separation of lead and lag sources.

\section{Method}

Subjects. Five of the 6 subjects from Experiment 1 participated in this experiment. The 6th, K.F., was unavailable. Thus, there were 2 males and 3 females, 23-38 years of age.

Apparatus, Stimuli, and Procedure. Testing chamber and stimuli were identical to those described for Experiment 1. Discrimination suppression thresholds and echo suppression thresholds were determined according to the adaptive procedures de- scribed for Experiment 1; however, thresholds were measured only for the baseline condition and not for the buildup condition.

In order to have a large range of azimuthal separations, a different loudspeaker configuration was employed from that employed in Experiment 1 (see Figure 5). The lag position was held constant at $+45^{\circ}$, and the lead loudspeaker was positioned at one of five azimuths $\left(-60^{\circ},-15^{\circ},+15^{\circ},+45^{\circ}\right.$, and $\left.+60^{\circ}\right)$. As described previously, in the echo suppression task, the lag loudspeaker was at $+45^{\circ}$, and the subject reported "one" or "two" events; in the discrimination suppression task, the lag loudspeaker was either at $+35^{\circ}$ or at $+55^{\circ}$, and the subject had to report which speaker delivered the echo. Within an adaptive block of trials, lead loudspeaker azimuth was held constant; the order of the 10 conditions ( 5 lead azimuths $\times 2$ types of suppression thresholds) was random and counterbalanced across subjects. At least four thresholds were obtained for each condition (using the same rules for inclusion as described for Experiment 1), and the final reported thresholds are the averages across all threshold runs completed for that condition.

\section{Results and Discussion}

Figure 6 presents the data for the 5 subjects individually as well as the mean data across the 5 subjects (in the lower right panel). In each panel, thresholds are plotted as a function of the lead source azimuth (the position of the lag source is indicated as an arrow on the abscissa). Echo suppression thresholds are shown as filled circles; discrimination suppression thresholds are shown as open triangles.

Lead-lag spatial separation exhibited no consistent effect on performance. A $2 \times 2$ ANOVA performed on the data, with azimuth $\left(-60^{\circ},-15^{\circ}, 15^{\circ}, 45^{\circ}\right.$, and $\left.60^{\circ}\right)$ and suppression (echo suppression and discrimination suppression) as within-subjects factors, indicated that neither of the main effects nor the interaction between them 


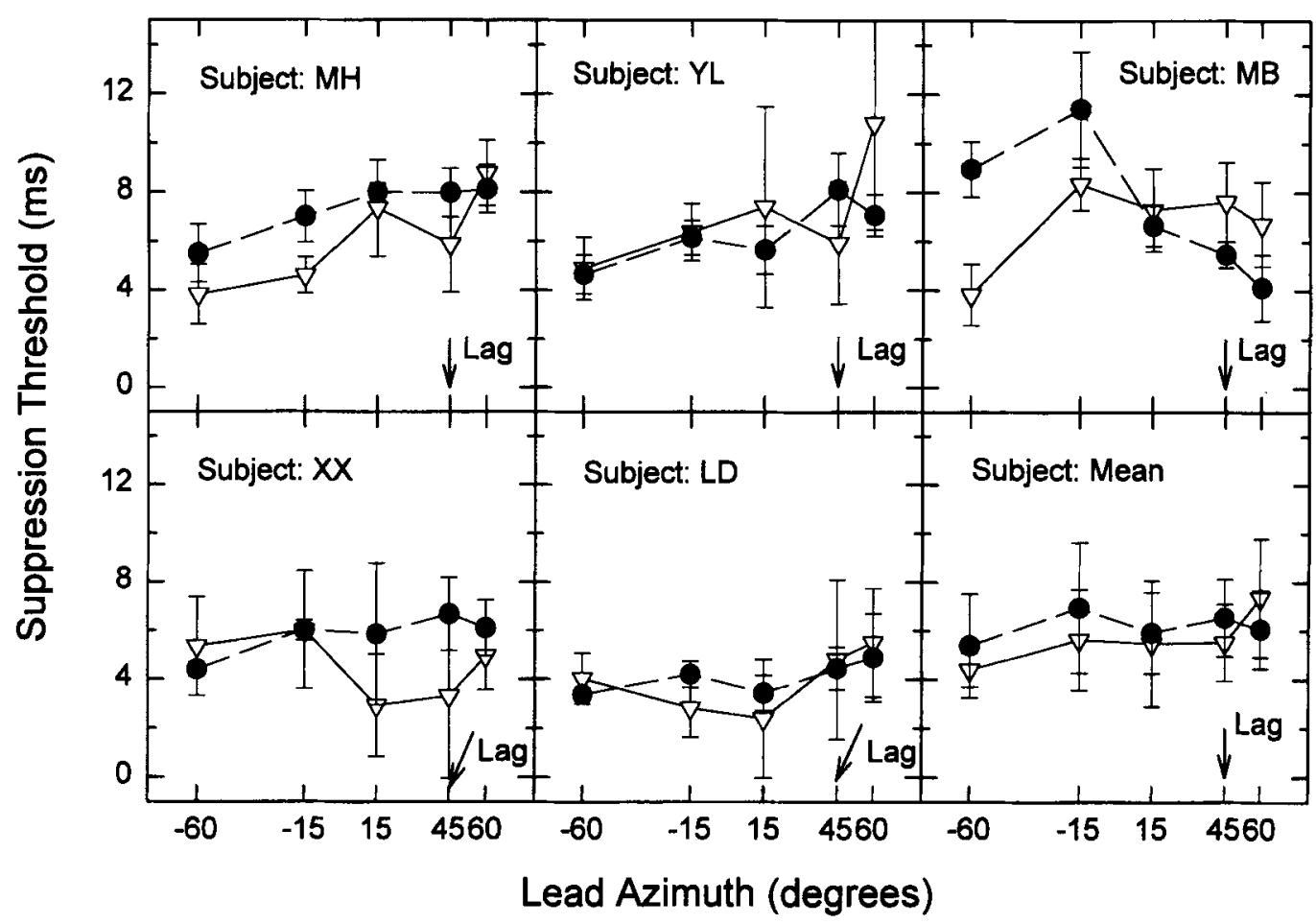

Figure 6. Echo suppression thresholds (filled circles) and discrimination suppression thresholds (open triangles) as a function of the location of the lead loudspeaker. Data are shown for the 5 subjects in different panels (means across subjects are shown in the lower right panel). The position of the lag loudspeaker is shown as the arrow along the abscissa in each panel. Error bars show \pm 1 standard deviation computed across runs for the individual subjects or computed across subjects for the mean data (in the lower right panel).

was significant. Follow-up tests revealed no significant effect of lead source azimuth on either echo suppression or discrimination suppression.

Large individual differences were apparent; however, within each subject, discrimination suppression and echo suppression functions had similar patterns. For example, Subjects M.H. and Y.L. showed increasing functions for both discrimination suppression and echo suppression, Subjects X.X. and L.D. showed rather flat functions, and Subject M.B. showed nonmonotonic functions. Overall, the results support the finding from Experiment 1 that discrimination suppression and echo suppression are mediated by a common mechanism for baseline (i.e., singlestimulus) presentation.

The results do not support the prediction that discrimination suppression would increase and echo suppression would decrease as lead and lag source positions diverge. This expectation was based on previous reports in the literature that had suggested this direction of results. One reason our results did not show the same pattern as those previously reported is that the earlier studies employed stimuli that might have produced some buildup effects. With regard to the previous reports that echo suppression decreases with increasing lead-lag separation, the Yost and Guzman (1995) study employed click trains, such as those employed in Experiment 1 of the present study, and was explicitly investigating buildup effects. ${ }^{5}$ Similarly, the one previous study that examined discrimination as a function of lead-lag separation employed two pairs of two clicks on each trial, and the total set of four clicks may well have produced buildup of suppression. On the basis of increasing evidence that buildup stimulation results in patterns of suppression that are significantly different from those of baseline stimulation (Experiment 1 in present study; Grantham, 1996), it is possible that the present negative result was due to testing only under baseline conditions, where any possible differential effects may have been too small to have been detected.

\section{SUMMARY AND CONCLUSIONS}

The precedence effect, whereby a direct sound and its echo are heard as a single sound coming from the vicinity of the lead sound, encompasses three aspects, represented by three types of tasks that have been reported in the literature. The present study investigated two of these aspects: (1) echo suppression (the direct sound and its echo are fused, and subjects can hear only one event), and (2) discrimination suppression (subjects cannot reliably discriminate the position of the echo). Echo suppression threshold and discrimination suppression threshold were relatively small for single burst-pair presentation ( $5 \mathrm{msec}$ in the present investigation). Under buildup con- 
ditions (when a train of redundant burst pairs preceded the test burst), both discrimination suppression threshold and echo suppression threshold increased; however, the amount of increase in echo suppression threshold $(21 \mathrm{msec})$ was substantially greater than that in discrimination suppression threshold $(5 \mathrm{msec})$. This result indicates that there is a range of echo delay for which the subject reports only a single event, but for which she or he is able to discriminate the echo's position at an abovechance level. The differential increase in threshold, together with the long time period involved in buildup, may be due to the involvement of more central processes in the buildup precedence effect, which serves to reduce subjects' awareness of the echoes produced in a familiar environment. These findings also suggest that mechanisms involved in the buildup of echo suppression may be different from those involved in the buildup of discrimination suppression.

Neither echo suppression nor discrimination suppression was dependent upon the azimuthal separation between lead and lag sources when measured in a baseline condition. However, on the basis of scattered results from previous studies, it is reasonable to expect that different results might be obtained if these measurements were made with buildup stimuli. In particular, previously cited data suggest that echo suppression threshold will decrease but that discrimination suppression threshold will increase as the echo's position moves away from that of the direct sound; in other words, the echo's position will become more difficult to discriminate with increasing lead-lag separation, even as it becomes more perceptible as a separate event. Whether these predictions will be borne out with buildup stimuli remains to be investigated.

\section{REFERENCES}

BlAUERT, J. (1983). Spatial hearing: The psychophysics of human sound localization (J. S. Allen, Trans.). Cambridge, MA: MIT Press. (Original work published 1974)

Clifton, R. K. (1987). Breakdown of echo suppression in the precedence effect. Journal of the Acoustical Society of America, 82, 1834-1835.

Clifton, R. K., \& Freyman, R. L. (1989). Effect of click rate and delay on breakdown of the precedence effect. Perception \& Psychophysics, 46, 139-145.

Clifton, R. K., Freyman, R. L., Litovsky, R. Y., \& McCall, D. (1994). Listeners' expectations about echoes can raise or lower echo threshold. Journal of the Acoustical Society of America, 95, 1525-1533.

Freyman, R. L., Clifton, R. K., \& Litovsky, R. Y. (1991). Dynamic processes in the precedence effect. Journal of the Acoustical Society of America, 90, 874-884.

Grantham, D. W. (1996). Left-right asymmetry in the buildup of echo suppression in normal-hearing adults. Journal of the Acoustical Society of America, 99, 1118-1123.

Hafter, E. R., Saberi, K., Jensen, E. R., \& Briolle, F. (1992). Localization in an echoic environment. In Y. Cazals, K. Horner, \& L. Demany (Eds.), Auditory physiology and perception (pp. 555-561). Oxford: Pergamon.

LEviTT, H. (1971). Transformed up-down methods in psychoacoustics. Journal of the Acoustical Society of America, 49, 467-477.

Litovsky, R. Y., Dizon, R., Pazmany, C., \& Colburn, H. S. (1996, February). Studies of the precedence effect in the median sagittal and azimuthal planes in a virtual acoustic space. Paper presented at the meeting of the Association for Research in Otolaryngology, Clearwater Beach, FL.

LitovsKy, R. Y., \& MACMILLAN, N. A. (1994). Sound localization precision under conditions of the precedence effect: Effects of azimuth and standard stimuli. Journal of the Acoustical Society of America, 96, 752-758.

Perrott, D. R., Marlborough, K., Merrill, P., \& Strybel, T. Z (1989). Minimum audible angle thresholds obtained under conditions in which the precedence effect is assumed to operate. Journal of the Acoustical Society of America, 85, 282-288.

RAKERD, B., \& HARTMANN, W. M. (1986). Localization of sound in rooms III: Onset and duration effects. Journal of the Acoustical Society of America, 80, 1695-1706.

SABERI, K., \& PERROTT, D. R. (1990). Lateralization thresholds obtained under conditions in which the precedence effect is assumed to operate. Journal of the Acoustical Society of America, 87, 1732-1737.

Shinn-Cunningham, B. G., Zurek, P. M., \& Durlach, N. I. (1993). Adjustment and discrimination measurements of the precedence effect. Journal of the Acoustical Society of America, 93, 2923-2932.

Thurlow, W. R., \& Parks, T. E. (1961). Precedence-suppression effects for two click sources. Perceptual \& Motor Skills, 13, 7-12.

Wallach, H., Newman, E. B., \& Rosenzweig, M. R. (1949). The precedence effect in sound localization. American Journal of Psychology, 62, 315-336.

YANG, X., \& GRANTHAM, D. W. (1993). Buildup of echo suppression: Effects of echo delay and source position [Abstract]. Journal of the Acoustical Society of America, 93, 2316.

YANG, X., \& GRanThaM, D. W. (1994). Effects of center frequency and bandwidth on echo threshold and buildup of echo suppression [Abstract]. Journal of the Acoustical Society of America, 95, 2917.

YANG, X., \& GRANTHAM, D. W. (1995). Localization suppression and echo suppression aspects of the precedence effect [A bstract]. Journal of the Acoustical Society of America, 97, 3280.

Yost, W. A., \& Guzman, S. J. (1995). Precedence and plausibility [Abstract]. Journal of the Acoustical Society of America, 97, 3412. YosT, W. A., \& SoDERQuIST, D. R. (1984). The precedence effect: Revisited. Journal of the Acoustical Society of America, 76, 1377-1383. ZUREK, P. M. (1980). The precedence effect and its possible role in the avoidance of interaural ambiguities. Journal of the Acoustical Society of America, 67, 952-964.

ZuREK, P. M. (1987). The precedence effect. In W. A. Yost \& G. Gourevitch (Eds.), Directional hearing (pp. 85-105). New York: Springer-Verlag.

\section{NOTES}

1. What we call discrimination suppression in the present paper was referred to as localization suppression in the published abstract describing some of the same data (Yang \& Grantham, 1995). We now believe the former term is more appropriate to describe the task employed (discriminating the location of the echo event); also, it is in concert with the terminology employed by Shinn-Cunningham et al. (1993).

2 . We reported some data at the 125 th Meeting of the Acoustical Society of America (Yang \& Grantham, 1993) on discrimination suppression. We found that discrimination suppression increased when the test click pair was preceded by a train of nine similar click pairs (this result replicated some of the findings of Freyman et al., 1991); furthermore, the amount of increase in discrimination suppression tended to be greater when the spatial and temporal characteristics of the test stimulus were the same as those of the preceding train than when they differed from those of the preceding train (this result is similar to some findings of Clifton et al., 1994). The following year, we reported some data on the effects of stimulus frequency and bandwidth on echo suppression (echo suppression threshold tended to increase as stimulus center frequency and/or bandwidth decreased; Yang \& Grantham, 1994). Two listeners were common to these two experiments, and we noticed that, for comparable stimulus conditions in the two studies, they showed consistently higher echo suppression thresh- 
olds than discrimination suppression thresholds. Subsequent informal listening confirmed this directional difference for cases when a test click was preceded by a train of similar clicks.

3. In this experiment, it was not possible to use the catch trials to estimate a false-alarm rate, since the subjects reported "one event" $100 \%$ of the time to the catch trials. For purposes of the present study, catch trials served to provide the subjects with a periodic exemplar of a single sound; even though the subjects' criterion could thus not be quantified, it was felt that the periodic presentation of an exemplar served to stabilize the subjects' criteria.

4. One of the reviewers of this paper suggested a clever modification of our experiment, which may produce a "purer" measure of the difference in echo suppression threshold and discrimination suppression threshold under buildup conditions than the measure we obtained. In the echo suppression experiment, the train would be presented nor- mally (as shown on the left side of Figure 2), but, for the test burst presentation, the lag burst would be presented from Loudspeaker 3 instead of the midline loudspeaker (Loudspeaker 1); the subject would, as before, respond "one" or "two." In this way, the responses in both the fusion and the discrimination tasks would be based on a loudspeaker position shifted $5^{\circ}$ from that employed during the train, and any "release from buildup" would thus be equivalent for the two tasks.

5 . The other two previously mentioned studies (Boerger, as reported in Blauert, 1983; Hafter et al., 1992) may have also effectively employed buildup stimuli, depending on such factors as the pace of stimulus presentation and duration of the stimulus on each presentation. These details were not evident from the respective papers.

(Manuscript received June 7, 1996; revision accepted for publication November 4, 1996.) 